\title{
Polydisperse hard spheres at a hard wall
}

\author{
Matteo Buzzacchi, ${ }^{1}$ Ignacio Pagonabarraga, ${ }^{2}$ and Nigel B. Wilding ${ }^{1}$ \\ ${ }^{1}$ Department of Physics, University of Bath, Bath BA2 7AY, United Kingdom \\ ${ }^{2}$ Universitat de Barcelona, Dept Fisica Fonamental, E-08028 Barcelona, Spain
}

(Dated: November 13, 2018)

\begin{abstract}
The structural properties of polydisperse hard spheres in the presence of a hard wall are investigated via Monte Carlo simulation and density functional theory (DFT). Attention is focussed on the local density distribution $\rho(\sigma, z)$, measuring the number density of particles of diameter $\sigma$ at a distance $z$ from the wall. The form of $\rho(\sigma, z)$ is obtained for bulk volume fractions $\eta_{b}=0.2$ and $\eta_{b}=0.4$ for two choices of the bulk parent distribution: a top-hat form, which we study for degrees of polydispersity $\delta=11.5 \%$ and $\delta=40.4 \%$, and a truncated Schulz form having $\delta=40.7 \%$. Excellent overall agreement is found between the DFT and simulation results, particularly at $\eta_{b}=0.2$. A detailed analysis of $\rho(\sigma, z)$ confirms the presence of oscillatory size segregation effects observed in a previous DFT study (Pagonabarraga et al., Phys. Rev. Lett. 84, 911 (2000)). For large $\delta$, the character of these oscillation is observed to depend strongly on the shape of the parent distribution. In the vicinity of the wall, attractive $\sigma$-dependent depletion interactions are found to greatly enhance the density of the largest particles. The local degree of polydispersity $\delta(z)$ is suppressed in this region, while further from the wall it exhibits oscillations.
\end{abstract}

\section{INTRODUCTION AND BACKGROUND}

Many complex fluids, whether natural or synthetic in origin, are intrinsically polydisperse in character, that is they comprise mixtures of similar rather than strictly identical constituent particles. Common examples are colloidal dispersions, macromolecules in solution and liquid crystals, where variation amongst the particles can occur in attributes such as their size, shape or surface charge. Polydispersity is of considerable practical relevance because it can affect the properties of materials in a variety of applications such as coating technologies [1], foodstuffs [2] and polymer processing [3]. However, from the fundamental perspective, the current understanding of polydisperse fluids is considerably less advanced than that of their monodisperse counterparts. The reason for this is the inherent complexity that polydispersity imparts to a system, and which generates difficulties for experimental, theoretical and simulation approaches alike.

On the experimental front, intricate technical issues beset the preparation, characterization and analysis of polydisperse samples, and only relatively recently has work begun to systematically address the generic consequences of polydispersity, such as its effects on phase behaviour and the fractionation of particles of different sizes between coexisting phases [4-6]. As far as analytical theory is concerned, the principal challenge is the multitude of variables required to accurately describe the system's properties. Statistical mechanical theories of polydispersity typically regard the polydisperse attribute as a continuous variable $(\sigma$, say) $[7,8]$. Accordingly the system may be regarded as a mixture of an infinite number of species-each labelled by the value of $\sigma$. It is then natural to describe the polydispersity in terms of a density distribution, $\rho^{0}(\sigma)$, measuring the number density of each species. Difficulties arise, however, when one attempts to determine the thermodynamic properties of the system, such as its phase behaviour. Because the free energy de- pends on (is a functional of) the entire distribution $\rho^{0}(\sigma)$, it occupies a parameter space that is effectively infinite dimensional. This in turn renders the analysis of phase behaviour much more problematic than for monodisperse systems or finite mixtures of a few components.

For small degrees of polydispersity, perturbative approaches permit some theoretical headway to be made $[9,10]$. A more general approach is the moment free energy (MFE) method [11-14]. For certain truncatable forms of the free energy use of this method allows the effective dimensionality of the problem to be reduced to a manageable level by projecting the free energy onto a suitably defined subspace spanned by a few principal moments of $\rho^{0}(\sigma)$. The phase behaviour of the resulting projected free energy can be calculated relatively straightforwardly. This method has been applied to investigate a range of open questions related to bulk phase equilibria, including (inter-alia) issues concerned with liquid-vapor coexistence and critical point shifts [15], freezing [16, 17] and liquid crystal phase transitions [18].

These advances in theoretical methods for dealing with polydisperse fluids, have recently been complemented by parallel developments in simulation methodologies. New Monte Carlo (MC) algorithms now permit the effective study of polydisperse phase equilibria within the grand canonical ensemble (GCE). Use of this ensemble is advantageous for the study of phase transitions because it allows the system density to fluctuate as a whole, thereby catering naturally for both order parameter fluctuations and fractionation effects [21]. Within the GCE framework, polydispersity is controlled by means of a chemical potential distribution $\mu(\sigma)$, the form of which is adapted (for each state point of interest) in such a way as to yield an ensemble averaged density distribution $\bar{\rho}(\sigma)$ that matches some prescribed form $\rho^{0}(\sigma)$. This approach extends previous ones (see eg. refs.[19, 20]) by facilitating the targeting of a specific density distribution. It thus corresponds more closely to the experi- 
mental situation for polydisperse fluids such as colloids and polymers, where the form of $\rho(\sigma)$ is fixed by the synthesis of the fluid and only its scale can vary depending on the quantity of solvent present. The new techniques have recently been applied to obtain the equation of state of polydisperse hard spheres [21] and to investigate polydispersity-induced alterations to bulk phase behaviour in a Lennard-Jones fluid [15].

While there is considerable ongoing progress in the study of bulk phase behaviour, to date comparatively little work has been reported regarding the influence of polydispersity on the properties of inhomogeneous fluids [22]. One of the standard contemporary theoretical approaches for dealing with such systems is density functional theory (DFT) [23]. Recently, it has been shown by one of us, that the MFE method outlined above carries over straightforwardly to certain well established density functionals $[24,25]$. The first such study of an inhomogeneous system considered the prototype model for a inhomogeneous polydisperse fluid, namely hard spheres in the vicinity of a hard wall [24]. Novel oscillatory behaviour was reported in the local concentration of different sized species as a function of the wall distance. Other DFT work has investigated polydisperse polymer/solvent mixtures at coexistence [25], and the effect of polydispersity on the fractionation and surface tension of a liquid-gas interface within a van der Waals approximation [26].

The extension of DFT techniques to polydisperse fluids is a welcome development. However, as yet, the reliability of the approximations inherent in such treatments remain untested. Clearly, therefore, it is desirable to obtain benchmarks with which these and other theories of confined polydisperse fluids can be compared. In this paper we address this issue using MC simulation and DFT applied to size-disperse hard spheres at a hard wall. For this purpose the GCE simulation methods developed for the study of the bulk phase equilibria of polydisperse fluids carry over directly, allowing us to probe confinementinduced changes to the density distribution. In addition to facilitating a detailed comparison between simulation and DFT, our results confirm the presence of the oscillatory size segregation effects seen previously in the original DFT study, as well as further elucidating the character of the local fluid structure and the effects of depletion interactions near the wall.

Our article is organized as follows: in Sec. II we outline the MC simulation scheme and the density functional theory. In Sec. III we analyse and compare in detail the MC and DFT results for two choices of the parent size distribution at two volume fractions. Finally, a discussion of the results and the prospects for interesting further work features in Sec. IV.

\section{SYSTEM AND METHODOLOGY}

The system with which we shall be concerned is a fluid of hard spheres, whose polydisperse attribute $\sigma$ corre- sponds to the sphere diameter. Pairs of particles $i$ and $j$ are assumed to interact via the potential

$$
U_{p p}\left(\mathbf{r}_{i}, \mathbf{r}_{j}, \sigma_{i}, \sigma_{j}\right)=\left\{\begin{array}{cl}
+\infty & \text { if }\left|\mathbf{r}_{i}-\mathbf{r}_{j}\right|<\frac{\sigma_{i}+\sigma_{j}}{2} \\
0 & \text { otherwise }
\end{array}\right.
$$

Consider initially such a system in the bulk. Conventionally the state of the system is described by a "parent" density distribution $[14,27] \rho^{0}(\sigma)$, which can be written

$$
\rho^{0}(\sigma)=n_{0} f(\sigma) .
$$

Here $n_{0}=N / V$ is the overall particle number density, while $V$ is the system volume and $f(\sigma)$ is a normalized shape function, the relative width of which is quantified by the dimensionless degree of polydispersity:

$$
\delta=\frac{\sqrt{\overline{(\sigma-\bar{\sigma})^{2}}}}{\bar{\sigma}},
$$

measuring the standard deviation of the parent distribution, normalized by its mean.

Let us now break the translational symmetry of the system in one coordinate direction ( $z$, say) by introducing a hard wall in the plane $z=0$. Interactions of the particles with the wall are assumed to be controlled by the potential:

$$
U_{p w}\left(z_{i}, \sigma_{i}\right)=\left\{\begin{array}{cl}
+\infty & \text { if } z_{i}<\sigma_{i} / 2 \\
0 & \text { otherwise }
\end{array}\right.
$$

Near such a wall, modifications to bulk behaviour arise even in monodisperse systems from standard packing effects. For polydisperse systems, however, additional factors come into play. Firstly, the excluded volume constraint associated with $U_{p w}$ implies that the centers of small particles are permitted to approach the wall more closely than those of large particles. Hence one expects (for sufficiently small $z$ ) that the local density distribution will be truncated at large $\sigma$ with respect to the bulk (parent) form. Secondly, experience with binary hard sphere mixtures [28] suggest that very close to the wall one can expect attractive depletion interactions between the particles and the wall. In the polydisperse case these depletion forces should be $\sigma$-dependent. In order to quantify the net influence of all these effects, we consider the ensemble averaged local density distribution $\rho(\sigma, z)$ at a perpendicular distance $z$ from the wall:

$$
\rho(\sigma, z)=\int_{0}^{L_{x}} \int_{0}^{L_{y}} \rho(\sigma, \mathbf{r}) d x d y .
$$

In the present work, we have elected to study two forms of the parent distribution: a top-hat distribution and a Schulz, the form of which are shown in fig. 1. The top-hat distribution is defined by 


$$
f_{t h}(\sigma)=\left\{\begin{array}{cl}
(2 c)^{-1} & \text { if } 1-c \leq \sigma \leq 1+c \\
0 & \text { otherwise }
\end{array},\right.
$$

where (without loss of generality), the mean particle diameter has been set to $\bar{\sigma}=1.0$. We have studied the cases $c=0.2$ and $c=0.7$, for which $\delta=c / \sqrt{3} \approx 0.115$ and $\delta \approx 0.404$ respectively.

The Schulz distribution is defined by the shape function

$$
f_{s z}(\sigma)=\frac{1}{z !}\left(\frac{z+1}{\bar{\sigma}}\right)^{z+1} \sigma^{z} \exp \left[-\left(\frac{z+1}{\bar{\sigma}}\right) \sigma\right] .
$$

Here the parameter $z$ controls the width of the distribution and thence the value of $\delta$. We have considered the case $z=5$, corresponding to $\delta=(z+1)^{-1 / 2} \simeq 0.408$. Again, by construction, $\bar{\sigma}=1.0$. Notice that in contrast to eg. a Gaussian, the Schulz distribution vanishes smoothly (has a natural cutoff) as $\sigma \rightarrow 0$. For the purposes of the MC simulations described below, however, one does require an upper cutoff in $\sigma$. We chose to truncate $f(\sigma)$ at $\sigma_{c}=4.0$, which reduces the degree of polydispersity to $\delta \approx 0.407$. The truncated form of $f(\sigma)$ was then rescaled to unit integrated area. Given this truncation, the population of particles at the cutoff diameter is very small compared to those having the mean diameter: $\rho^{0}\left(\sigma_{c}\right) / \rho^{0}(\bar{\sigma})=1.7 \times 10^{-5}$. However, the volume of the largest allowed particle is 64 times that of one having the mean diameter.

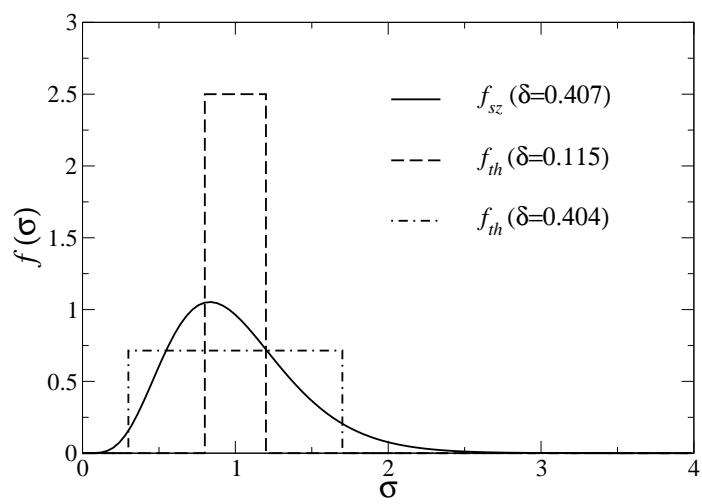

FIG. 1: The top-hat and Schulz distributions considered in this work.

A quantity useful in characterizing a polydisperse system is its volume fraction, which provides a measure of the degree to which the particles pack the available space. We shall employ it in preference to the number density $n_{0}$ which does not alone provide equivalent information. We define the volume fraction in terms of the bulk parent distribution:

$$
\eta_{b}=\frac{\pi}{6} \int d \sigma \rho^{0}(\sigma) \sigma^{3}
$$

For a nominated shape function $f(\sigma)$, choosing a value for $\eta_{b}$ (or equivalently $n_{0}$ ) serves to fix the bulk chemical potential distribution $\mu(\sigma)$. The assumption that the confined fluid exists in equilibrium with a bulk reservoir then implies that both the bulk and confined systems have equal $\mu(\sigma)$. Note however, that for the confined hard sphere systems we consider here, the system-averaged volume fraction is generally less than that of the reservoir. For both the top-hat and Schulz parents described above, we consider the cases $\eta_{b}=0.2$ and $\eta_{b}=0.4$.

With regard to our use of scales, we shall throughout express all lengths in units of the average particle diameter $\bar{\sigma}$ of the parent distribution. Owing to the lack of a temperature scale in hard sphere systems, we implicitly adopt the convention of assigning $\beta=1 / k_{B} T \equiv 1$.

\section{A. Grand-canonical ensemble Monte Carlo calculations}

The Grand Canonical Metropolis Monte Carlo algorithm we have employed has previously been described in ref. [21] and involves particle insertions, deletions, translations, and resizing operations. Particle resizing operations $\left(\sigma \rightarrow \sigma^{\prime}\right)$, or the insertion of a new particle are performed by drawing a random number with uniform probability in the range of allowed sizes. Acceptance/rejection criteria for these moves involve not only the change in the particle-particle and particle-wall potentials, but also the change in the chemical potentials $\mu(\sigma)$ and $\mu\left(\sigma^{\prime}\right)$. Translational moves $(\sigma, \mathbf{r}) \rightarrow\left(\sigma, \mathbf{r}^{\prime}\right)$ are not strictly necessary in the GCE formalism, as they are equivalent to the successive deletion of particle $(\sigma, \mathbf{r})$ and insertion of particle $\left(\sigma, \mathbf{r}^{\prime}\right)$, but their use proved beneficial to the sampling efficiency at the higher local volume fractions encountered near the wall.

Within the GCE framework, the form of the ensemble averaged density distribution $\bar{\rho}(\sigma)$ is controlled by its conjugate chemical potential distribution $\mu(\sigma)$. In order to perform simulations of a prescribed parent distribution one therefore needs to match $\bar{\rho}(\sigma)$ to $\rho^{0}(\sigma)$ in a bulk (or, in our case, fully periodic) simulation. Unfortunately, the task of determining the requisite $\mu(\sigma)$ is complicated by the fact that it is a functional of $\rho^{0}(\sigma)$. Effectively therefore, one is faced with solving the inverse problem $\mu(\sigma)=\mu\left[\rho^{0}(\sigma)\right]$. Doing so is facilitated by a recently proposed MC scheme-the non-equilibrium potential refinement (NEPR) algorithm [29]- use of which enables the efficient iterative determination of $\mu\left[\rho^{\circ}(\sigma)\right]$, from a single simulation run, and without the need for an initial guess of its form. To achieve this, the method continually updates $\mu(\sigma)$ in such a way as to correct for the deviation of the instantaneous density distribution $\rho(\sigma)$ from the target form (i.e. the parent). However, tuning $\mu(\sigma)$ in this manner clearly violates detailed balance. To counter this, successive iterations reduce the degree of modification applied to $\mu(\sigma)$, thereby driving the system towards equilibrium and ultimately yielding the equilibrium form 
of $\mu\left[\rho^{\circ}(\sigma)\right]$.

In order to obtain the bulk form of $\mu(\sigma)$ corresponding to the top-hat and Schulz distributions at $\eta_{b}=0.2$ and $\eta_{b}=0.4$, the NEPR algorithm was deployed in simulations of a fully periodic cubic simulation cell of side $L=12$. The resulting forms of $\mu(\sigma)$ for the respective parents were then used to study the effects of introducing two oppositely facing hard walls at $z=0$ and $z=L$ (the system remaining periodic in the $x$ and $y$ directions). The cell size $L=12$ proved sufficient to ensure that the fluid properties at the cell midpoint were indistinguishable (to within statistical uncertainty) from those of the fully periodic system. This finding was further tested by comparing the results with those for a cell that was elongated along the $z$ direction, having $\left(L_{z}=24\right)$. Again within statistical uncertainties, no differences were observed, leading us to conclude that the two walls do not interact with one another and that the properties near one wall are representative of the semi-infinite geometry.

The particle density $\rho(\sigma, z)$ was accumulated in the form of a histogram in the course of the simulations and will be the object of our analysis in the next section. The histogram was formed by discretising the permitted ranges $0 \leq \sigma \leq \sigma_{c}$ and $0<z<L_{z}$, into bins. The bin width in $\sigma$ used for the top-hat distributions was $\delta \sigma=0.01$, while $\delta \sigma=0.04$ was employed for the Schulz. The bin width in the $z$ direction was $\delta z=0.005$ for the top-hat distributions and $\delta z=0.02$ for the Schulz distribution.

\section{B. Density Functional Theory model}

The density functional model employed is that introduced by Rosenfeld [23, 30], following the implementation proposed in ref. [31], and corresponds to a weighted functional that has proved accurate for monodisperse systems [32]. Although improvements have been proposed to the original Rosenfeld model which appear to perform better in the context of describing crystalline phases, we do not expect our implementation to suffer major drawbacks for the regimes of volume fraction regimes of interest in the present work.

The free energy functional can be expressed as:

$$
\begin{aligned}
\Omega & =\int d \mathbf{r} d \sigma\left\{\rho(\sigma, \mathbf{r})\left[\ln \left(\Lambda^{3}(\sigma) \rho(\sigma, \mathbf{r})\right)-1\right]\right. \\
& +[V(\sigma, \mathbf{r})-\mu(\sigma)] \rho(\sigma, \mathbf{r})\}+\int d \mathbf{r} \mathcal{F}^{e x}\left[m_{\alpha}(\mathbf{r})\right]
\end{aligned}
$$

where the excess contribution to the free energy functional, $\mathcal{F}^{e x}=-m_{0} \ln \left(1-m_{3}\right)+m_{1} m_{2} /\left(1-m_{3}\right)+m_{2}^{3} /(1-$ $\left.m_{3}\right)^{2}$, is a function of four moments only:

$$
m_{\alpha}(\mathbf{r})=\int d \sigma d \mathbf{r}^{\prime} \rho(\sigma, \mathbf{r}) \omega_{\alpha}\left(\sigma,\left|\mathbf{r}-\mathbf{r}^{\prime}\right|\right)
$$

The four weight functions, $\omega_{\alpha}$ (non-local in space) are chosen to recover the Percus-Yevick free energy and correlation functions for a homogeneous mixture. In the bulk the moments have a simple physical interpretation. Indeed, $m_{3}(\infty)=\eta_{b}, m_{0}(\infty)=\rho_{b}$, while $m_{2}(\infty) / \rho_{b}$ is the mean area and $m_{1}(\infty) / \rho_{b}$ the mean radius.

The equilibrium density profiles are obtained by minimizing the free energy functional, eq.(9), with respect to the density profiles, leading to

$$
\rho_{e q}(\sigma, \mathbf{r})=\rho^{0}(\sigma) \exp \left[-\beta V(\sigma, \mathbf{r})+\mu^{e x}(\sigma, \mathbf{r})-\mu_{b}^{e x}(\sigma)\right]
$$

where $V(\sigma, \mathbf{r})$ accounts for an external potential, and $\mu_{b}^{e x}(\sigma)$ and $\rho^{0}(\sigma)$ correspond to the excess chemical potential and density of species $\sigma$ in the reservoir, respectively.

The advantage of the moment structure of the functional is that the excess chemical potential of each species is

$$
\begin{aligned}
\mu^{e x}(\sigma, \mathbf{r}) & =\sum_{\beta} \int d \mathbf{r} \omega_{\beta}\left(\sigma,\left|\mathbf{r}-\mathbf{r}^{\prime}\right|\right) \frac{\partial \mathcal{F}^{e x}}{\partial m_{\beta}}\left(\mathbf{r}^{\prime}\right) \\
& \equiv \sum_{\beta} \int d \mathbf{r} \omega_{\beta}\left(\sigma,\left|\mathbf{r}-\mathbf{r}^{\prime}\right|\right) \mu_{\beta}^{e x}\left(\mathbf{r}^{\prime}\right)
\end{aligned}
$$

and can be interpreted as a linear combination of the four moment excess chemical potentials, $\mu_{\alpha}^{e x}=\partial \mathcal{F}^{e x} / \partial m_{\alpha}$. This moment structure simplifies the study of polydisperse mixtures [14].

For the case of a fluid mixture in the presence of a planar hard wall, the external potential reduces to eq.(4): $V(\sigma, \mathbf{r})=U_{p w}(z, \sigma)$. The equilibrium density profiles, as given implicitly in eq. (11), are found numerically via iteration. To this end, it is necessary to define an underlying lattice and to represent the parent distribution as a set of discrete species. However, the existence of this lattice imposes a minimum resolvable length scale (lattice cutoff). To avoid lattice artifacts, one must therefore apply a lower cutoff to the parent distribution $\rho^{0}(\sigma)$ which is itself large compared to the lattice spacing; we take the lower $\sigma$ cutoff to vary between 20 and 50 lattice units. Although in principle, this cutoff may lead to discrepancies between the DFT and MC results (in the simulations particles can have a vanishingly small radius), we have verified that the lower cutoff is sufficiently small that changes to its value have negligible effect on the overall results.

Depending on the degree of polydispersity, the continuous parent distribution is represented by a few hundred species, ensuring always that the maximum sized species has a sufficiently small contribution to avoid spurious effects from the upper $\sigma$ cutoff. For consistency, we choose the upper cutoff to equal that used in the simulations. The mean particle diameter is always taken as the reference length scale in the calculations. 


\section{RESULTS}

Below we present our findings for the effect of the hard wall on the density distribution. For the purposes of gauging the accuracy of the DFT predictions, we have confined our comparison to the (representative) choice of the narrow top-hat distribution $(c=0.2, \delta=0.115)$ and the Schulz distribution $(z=5, \delta=0.407)$. Results for the wide top-hat $(c=0.7)$ have been obtained only via simulation, but serve to elucidate the effect of distribution shape changes at a given $\delta$. (a)

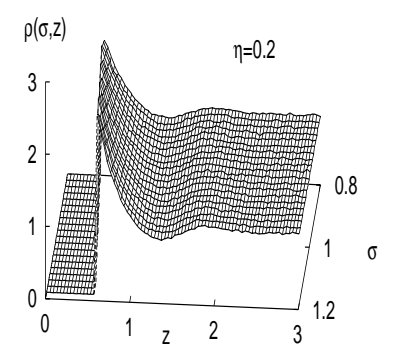

$\rho(\sigma, z)$

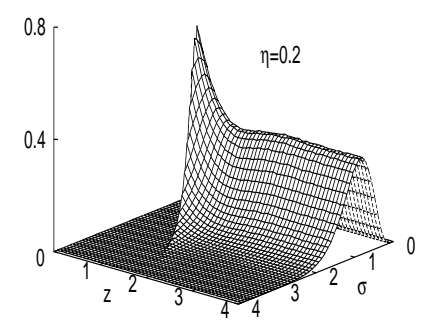

(b)

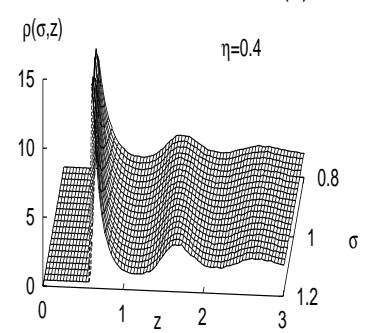

(d)

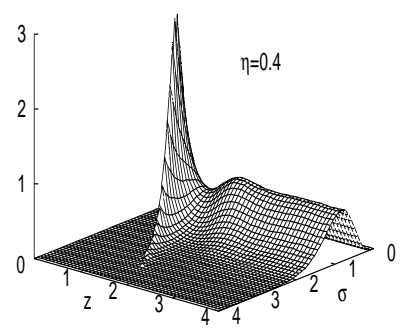

FIG. 2: The particle density distributions $\rho(\sigma, z)$ obtained from the MC simulations. (a,b) Top-hat $(\delta=0.115),(\mathbf{c}, \mathbf{d})$ Schulz.

An initial impression of the effects of confinement on the size distribution can be gained from fig. 2, which shows the form of $\rho(\sigma, z)$ obtained in the simulations for the narrow top hat and the Schulz distributions. Density oscillations normal to a solid interface are a common feature of confined fluids, and generally increase in magnitude with increasing volume fraction. In the polydisperse case, however, fig. 2 shows that the character of the oscillations can depend both on the particular choice of parent distribution and (for a given parent) on the value of $\sigma$.

In order to analyse the data contained in fig. 2 in greater detail, it is instructive to examine orthogonal slices $\rho(z \mid \sigma)$ and $\rho(\sigma \mid z)$. Beginning with the former case, examples of $\rho(z)$ for a selection of values of the particle
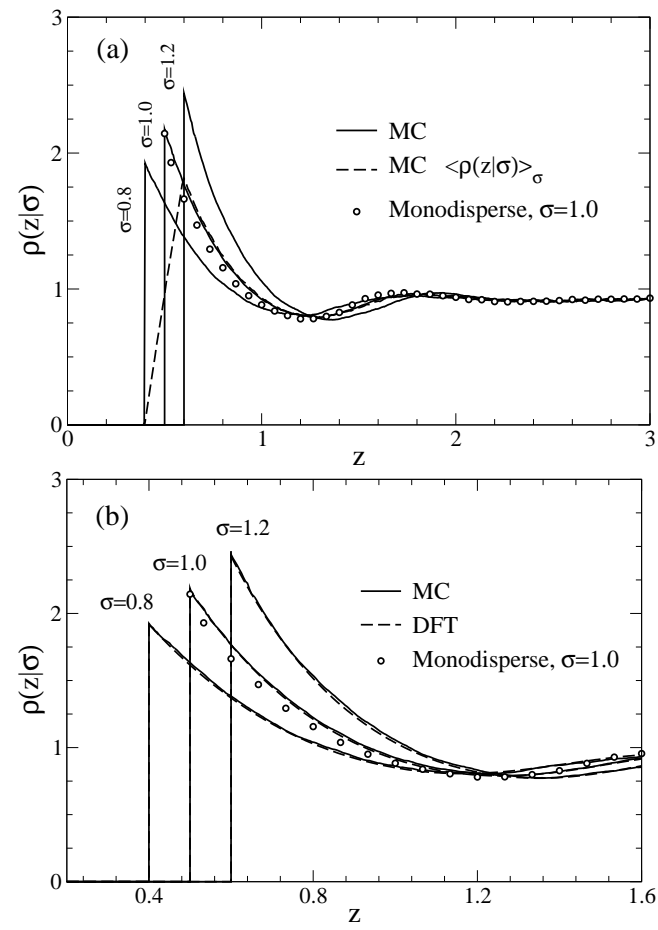

FIG. 3: (a) The spatial distribution $\rho(z \mid \sigma)$ for selected values of $\sigma$ for the top-hat distribution $(\delta=0.115)$ at $\eta_{b}=0.2$. (b) Detail showing the comparison between MC and DFT results in the region close to the wall.

diameter $\sigma$ are shown for the narrow top-hat distribution at $\eta_{b}=0.2$ and $\eta_{b}=0.4$ in figs. 3 and 4 respectively. The corresponding data for the Schulz distribution is shown in figs. 5 and 6 . Included also in these figures are the predictions of the DFT theory (cf. sec. IIB). In all cases one observes the presence of density oscillations which start from the bulk density value far from the wall and grow with decreasing $z$ down to the point $z=\sigma / 2$, where the curves terminate abruptly due to the excluded volume constraint of the hard wall. Also shown for comparison in each case is the form of $\rho(z)$ for monodisperse hard spheres (with $\sigma=1.0$ ) at the same respective volume fraction and normalized so that its value at $z=L / 2$ matches the density profile of the $\sigma=1.0$ species in the polydisperse system. Additionally, we provide the $\sigma$-averaged particle number density $\langle\rho(z \mid \sigma)\rangle_{\sigma}$ as well as (in cases where it deviates appreciable from the latter), the local volume fraction $\eta(z)=\frac{\pi}{6} \int d \sigma \rho(z \mid \sigma) \sigma^{3}$.

With regard to the general features of the profiles shown in figs, $3-6$, we note that an aspect peculiar to the narrow top-hat parent distribution is that $\rho(z)$ oscillates with a period $\sigma \simeq 1.0$ (i.e. close to $\bar{\sigma}$ ) for all particle sizes. This contrasts with the case of the much wider Schulz distribution, where the oscillations do not appear to be controlled by the average particle size, having a considerably larger wavelength in $z$. We note further that in all cases studied, the oscillations in $\rho(z)$ induced by the wall are more pronounced for the monodisperse fluid than for 

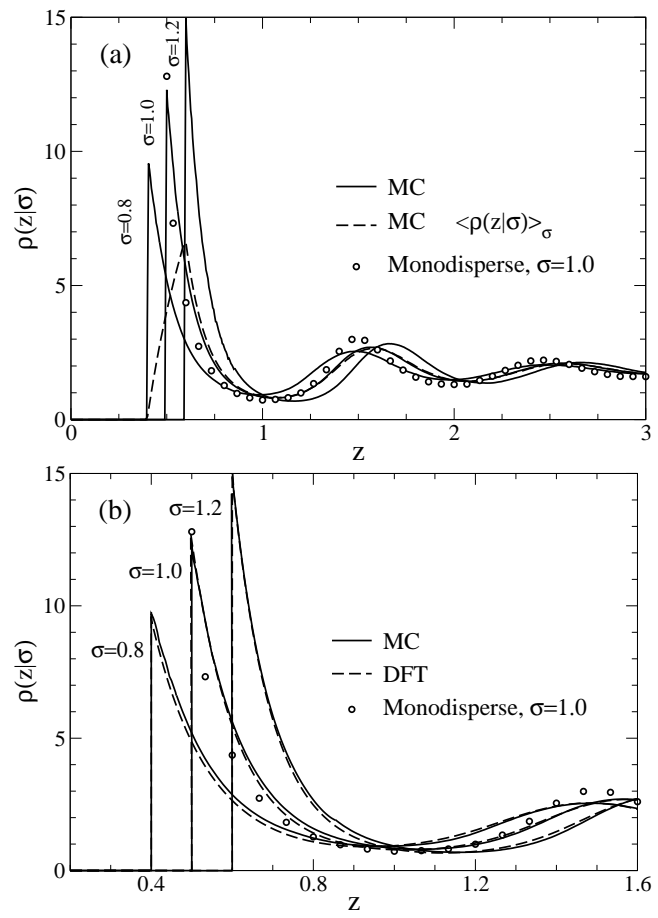

FIG. 4: (a) The spatial distribution $\rho(z \mid \sigma)$ for selected values of the particle size for the top-hat distribution $(\delta=0.115)$ at $\eta_{b}=0.4$. (b) Detail showing the comparison between MC and DFT results in the region close to the wall.

the polydisperse ones. Thus the effect of polydispersity appears to be to dampen the profile oscillations. This is presumably a result of structural disordering effects deriving from the ability of small particles to occupy the gaps between large ones. These in turn serve to disrupt the excluded volume packing effects which underly the density oscillations commonly observed in monodisperse systems near a hard wall. Within this qualitative picture, one would thus expect a greater dampening of the oscillations for the Schulz distribution than for the narrow top-hat, on account of the disparity in their values of $\delta$. This indeed appears to be the case, with the top-hat profiles resembling more closely the monodisperse case.

Turning next to the comparison of the simulation measurements with the results of the DFT calculations, it is gratifying to note that the level of agreement is generally excellent, particularly at the lower volume fraction. Only for the case $\eta_{b}=0.4$ and points close to the wall do we observe relatively minor systematic deviations, namely a slightly higher local density for the MC results.

As noted above, the profiles $\rho(z \mid \sigma)$ are truncated at $z=\frac{\sigma}{2}$ reflecting the geometrical constraint associated with the impenetrable wall at $z=0$. The density at this point, $\rho_{c}(\sigma) \equiv \rho\left(z=\frac{\sigma}{2} \mid \sigma\right)$ is the so-called contact value. In fig. 7 we plot $\rho_{c}(\sigma)$ for selected $\sigma$ for both the narrow and wide top-hat, as well as the Schulz distribution. From the figure, it is apparent that for both top-hat distributions, $\rho_{c}(\sigma)$ increases as a function of $\sigma$, i.e. the largest particles are preferentially favored over
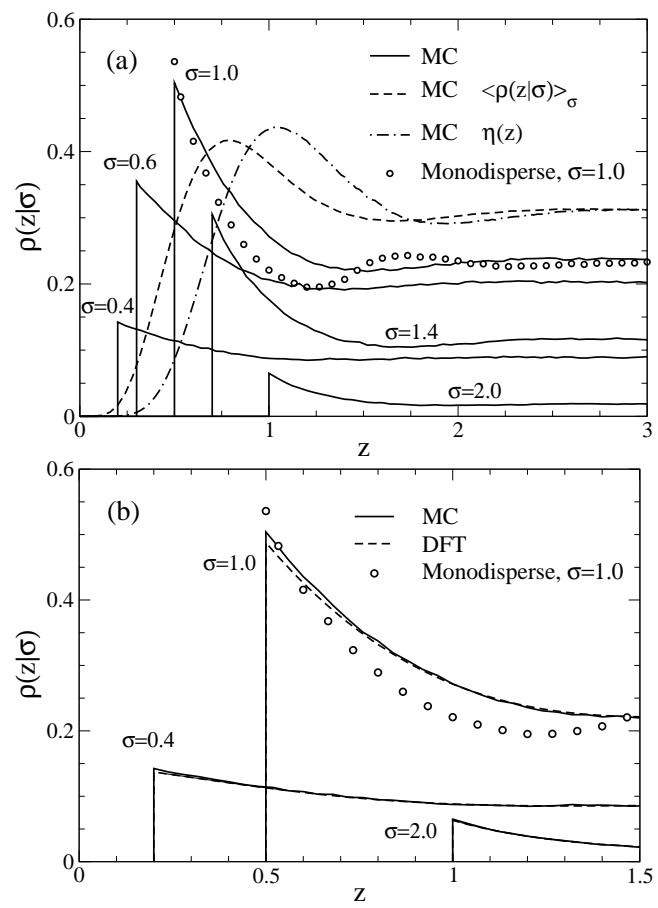

FIG. 5: (a) The spatial distribution $\rho(z \mid \sigma)$ for selected values of the particle size for the Schulz distribution at $\eta_{b}=0.2$. (b) Detail showing the comparison between MC and DFT results in the region close to the wall.

small ones at the wall. A similar effect occurs for the Schulz distribution, fig. 7(b), although in this case since the parent distribution is not constant in $\sigma$, one must form $\rho(z=\sigma / 2 \mid \sigma) / \rho^{0}(\sigma)$ in order to expose the density enhancement at contact (see fig. 7(c)).

The contact values are related to the partial pressures and hence the total pressure via the sum rule [33]:

$$
\beta p=\int d \sigma \rho_{c}(\sigma)
$$

The resulting estimates of the pressure are presented in table I where they are compared with the predictions of the moment based expressions of Mansoori et al (BMCSL) $[34,35]$ and Salacuse and Stell [8]. The former is based on a generalization to mixtures of the CarnahanStarling equation of state, while the latter is a generalization of the Percus-Yevick (PY) theory. The simulation estimates show good agreement with the analytical equations of state at the lower volume fraction $\eta_{b}=0.2$, though at the higher value there are discrepancies, with the BMCSL equation apparently predicting the observed pressure more accurately for the top-hat distribution and the PY equation faring better for the Schulz distribution. The latter result is in accord with previous simulation studies of the bulk equation of state for Schulz distributed hard spheres [21]. As regards the estimates for the pressure derived from the DFT contact values, we note the close agreement of these with the PY equation of 

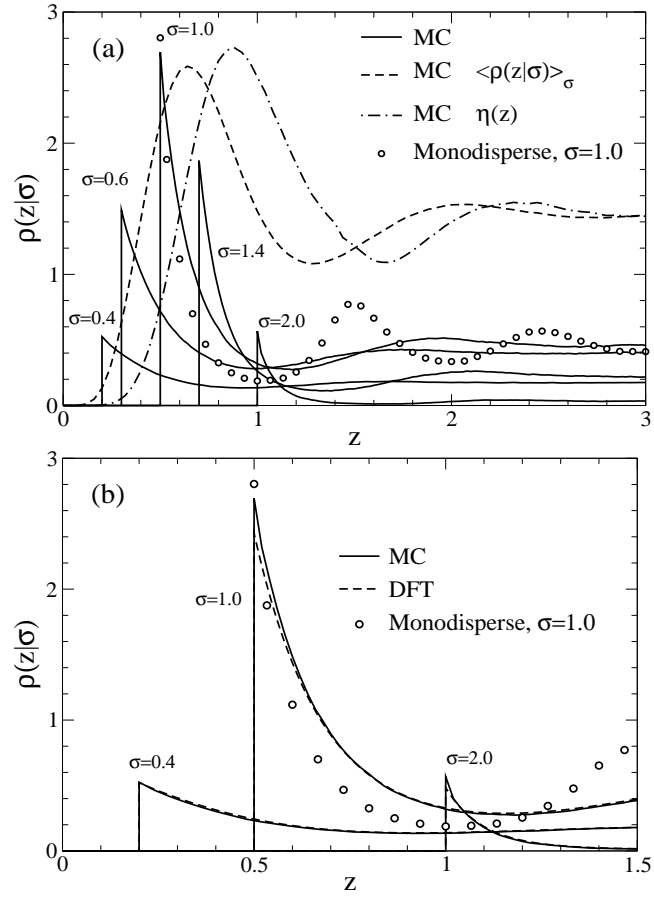

FIG. 6: (a) The spatial distribution $\rho(z \mid \sigma)$ for selected values of the particle size for the Schulz distribution at $\eta_{b}=0.4$. (b) Detail showing the comparison between MC and DFT results in the region close to the wall.

state. This finding is, however, not too surprising given that the specific Rosenfeld functional we employ is tuned to recover the PY equation in the bulk (see Sec. II B).

The enhancement (relative to the bulk) of the larger particles at contact (see fig. $7(\mathrm{c})$ ), is attributable to attractive depletion forces. It is interesting to note that for a given $\eta_{b}, \rho(z=\sigma / 2 \mid \sigma) / \rho^{0}(\sigma)$ is apparently very similar for the Schulz distribution and the wider of the two tophat parents. Since both these distributions have a very similar value of $\delta$, this suggests that the depletion interactions at the wall for a given $\sigma$ are primarily controlled by the values of $\eta_{b}$ and $\delta$, and are much less sensitive to the overall distribution shape.

The wall-fluid depletion interaction can be further quantified (see for example ref. [36]) in terms of an effective potential (potential of mean force) via:

$$
\frac{\rho(z \mid \sigma)}{\rho^{0}(\sigma)}=\exp \left(-\beta \Phi_{e f f}(z \mid \sigma)\right) .
$$

Here $\Phi_{\text {eff }}(z \mid \sigma)$ represents the excess grand potential of a particle of size $\sigma$ at distance $z$ from the wall with respect to its value in the bulk. Plots of $\Phi_{\text {eff }}(z \mid \sigma)$ (fig. 8) indeed show the expected attractive nature of the effective potential at contact, and the deepening of the well depth with increasing $\sigma$. Note, however, that the effective potential $\Phi_{\text {eff }}$ defined in eq.(14) should not be confused with a true two-body depletion potential. A relationship similar to eq.(14) has been used to quantify fluid-wall de- pletion effects in asymmetrical binary mixtures comprising large (colloid) particles in a solvent of small particles (such as polymer). There one defines a depletion potential between large particles and the wall in the limit in which the concentration of the large particles $[28,36]$ vanishes, but at fixed concentration of the smaller ones. By contrast, in the present case, the depletion forces are not mediated by a separate solvent but arise from the effects of the distribution of particle sizes [37]. Nevertheless similarities are evident with the form of the true two-body depletion potential observed in binary mixtures $[28,36]$. We note further, that the potentials for the Schulz distribution $(\delta=0.407)$ are numerically very similar to those for the wide top hat $(\delta=0.404)$ for distances $z \lesssim 2.0$. This suggests that our observation (see above) concerning the apparent insensitivity of the contact enhancements to the shape of $f(\sigma)$, extends to the form of the effective potential at significant distances away from the wall.

We turn next to an analysis of the distribution of particle sizes as a function of the distance $z$ from the wall, as expressed through the form of $\rho(\sigma \mid z)$. In figs. 9 and 10 , we plot $\rho(\sigma)$ at selected values of $z$ for both the narrow top-hat and Schulz parent distributions at bulk volume fractions $\eta_{b}=0.2$ and $\eta_{b}=0.4$. Focusing first on the top-hat case, one observes that as $z$ is increased (starting from near the wall), the form of $\rho(\sigma)$ alternates between being monotonically increasing and monotonically decreasing. This implies that with increasing $z$ the biggest and smallest particles are alternately favored, an effect known as local size segregation [24] (see also below). We note that at the closest allowed distance from the wall, large particles are always strongly favored. For large $z, \rho(\sigma)$ of course approaches the flat top-hat parent form.

In contrast to the case of the narrow top-hat, for the Schulz parent the density of the largest permitted particles (i.e. those allowed by the geometrical constraint at a given $z$ ) always exceeds the bulk density at that $\sigma$ (cf. fig. 10). The degree of enhancement is considerably stronger for $\eta_{b}=0.4$ than $\eta_{b}=0.2$. In both cases, for sufficiently small $z$, the distribution increases monotonically. However, as $z$ becomes larger, the enhancement in the density of the largest particles gradually reduces, and a peak (associated with the maximum of $\rho^{0}(\sigma)$ ) starts to appear at $z \simeq 1.0$, signalling the crossover to bulk behaviour. It is interesting to note that for $\eta_{b}=0.4$, the distribution for $1.05<z<\sigma_{c}$ is very broad and exhibits two maxima. One of these is related to the maximum of the parent distribution while the other corresponds to the depletion interaction-induced enhancement of the density of the largest particles permitted at that $z$. Again, we find that for both parent forms the DFT appears to capture semi-quantitatively the behaviour observed in the simulations with some relatively slight discrepancies to be found only at $\eta_{b}=0.4$.

In order to analyze further the local size segregation effects identified above, we have considered the spatial dependence of the local relative concentration defined as: 


\begin{tabular}{|c|cccc|}
\hline \hline Parent & MC & DFT & BMCSL & PY \\
\hline top-hat $\left(c=0.2, \eta_{b}=0.2\right)$ & $0.8718 \pm 0.008$ & 0.8748 & 0.8774 & 0.8774 \\
top-hat $\left(c=0.2, \eta_{b}=0.4\right)$ & $4.955 \pm 0.074$ & 5.074 & 4.971 & 5.180 \\
top-hat $\left(c=0.7, \eta_{b}=0.4\right)$ & $2.809 \pm 0.085$ & & 2.86 & 2.966 \\
Schulz $\left(\eta_{b}=0.2\right)$ & $0.518 \pm 0.009$ & & 0.5149 & 0.5175 \\
& & 0.516 & $(0.5147)$ & $(0.5173)$ \\
Schulz $\left(\eta_{b}=0.4\right)$ & $2.767 \pm 0.080$ & & 2.668 & 2.763 \\
& & 2.732 & $(2.593)$ & $(2.762)$ \\
\hline \hline
\end{tabular}

TABLE I: Estimates of the pressure $\beta p$, as calculated from the contact values (eq. 13) for the MC and DFT calculations for the top-hat and Schulz parents at the given volume fraction $\eta_{b}$. Also shown for comparison are the predictions of the BMCSL [34] and PY [8] equations of state. The values in brackets refer to the BMCSL and PY predictions for a Schulz parent having a lower cutoff in $\sigma$ as employed in the DFT calculations (see sec. II B).

$$
\phi(z, \sigma)=\frac{\rho(z, \sigma)}{\int_{0}^{\infty} d \sigma^{\prime} \rho\left(z, \sigma^{\prime}\right)}
$$

This quantity measures the concentration of species of size $\sigma$ at a given $z$. Size segregation is signalled by the appearance of an oscillatory structure in $\phi(z \mid \sigma)$. We have obtained the form of $\phi(z, \sigma)$ for both top-hat distributions (figs. 11,12) and the Schulz distribution (figs. 13). The oscillations are more pronounced at the larger $\eta_{b}$. For the narrow top-hat, a phase difference of approximately $\pi$ radians is observed between the largest and smallest permitted particles and the period is close to the average particle size [38]. However, for the wider top hat, the degree of anti-correlation is somewhat less and the period is extended. For the Schulz parent, comparison of the curves for $\phi(z \mid \sigma)$ is complicated by the fact that at large $z$ each curve converges to a limit that is proportional to $\rho^{0}(\sigma)$. Nevertheless, for a given $\sigma$, clear oscillations are visible, although they die out more rapidly than for the top hat and do not exhibit a well-defined anti-correlation.

Finally in this section we turn to our results for the local polydispersity $\delta(z)$ defined via a generalization of eq. 3 :

$$
\delta(z)=\frac{\left[\int d \sigma(\sigma-\bar{\sigma})^{2} \rho(\sigma \mid z)\right]^{1 / 2}}{\int d \sigma \sigma \rho(\sigma \mid z)} .
$$

Our measurements of this quantity for both top-hat parents and the Schulz parent are presented in fig. 14. One observes oscillations in $\delta(z)$, derived (cf. eq.16) from oscillations in both the local mean and the standard deviation of the density distribution. The oscillations are stronger for the wider parents than for the narrow one. Further insight into this behaviour can be gained from a perturbative analysis of $\delta(z)$ in the limit of a narrow parent. We assume that the species deviate slightly from the mean species, so that $\sigma=\bar{\sigma}(1+\epsilon)$ with $\epsilon$ a small parameter. In this limit, the density profile can be expressed $[24]$ as

$$
\begin{aligned}
\rho(\sigma, z) & =\frac{\rho(\bar{\sigma}, z) \rho^{0}(\sigma)}{\rho(\bar{\sigma}, \infty)} e^{-\beta[V(\sigma, z)-V(\bar{\sigma}, z)]} \\
& \times\left\{1+\epsilon\left[\tilde{c}^{\prime}(z)-\tilde{c}^{\prime}(\infty)\right]\right\}
\end{aligned}
$$

where $\rho(\bar{\sigma}, z)$ is the density profile for the mean species, while $\tilde{c}^{\prime}$ corresponds to the reversible work associated with slightly changing the size of one particle, a distance $z$ from the wall, within an otherwise monodisperse systems at the corresponding volume fraction (see [24] for further details). Introducing this expansion into eq.(16) yields

$$
\begin{aligned}
\delta(z) & \simeq \bar{\sigma} \sqrt{\frac{\rho(\bar{\sigma}, \infty) \overline{\epsilon^{2}}}{\rho(\bar{\sigma}, z) n_{0}}} \\
& \times\left\{1+\left[\overline{\frac{\epsilon^{3}}{2 \bar{\epsilon}^{2}}}-\overline{\epsilon^{2}}\right]\left[\tilde{c}^{\prime}(z)-\tilde{c}^{\prime}(\infty)\right]\right\},
\end{aligned}
$$

at distances where the wall potential is negligible.

Eq. 18 indicates that for a narrow parent the local deviations in the width of the distribution depend on second and higher moments of the parent. As a result, in this limit the local variations of the mean density make a relevant contribution to $\delta$. This is confirmed by fig. 14(a) which shows that the oscillations are indeed anticorrelated with those of the mean species. In fact, the amplitude of the term $\tilde{c}^{\prime}(z)-\tilde{c}^{\prime}(\infty)$ is around 0.004 for a flat parent with $\delta=0.115$ and around 0.054 for $\delta=0.404$. Since the deviations of $\tilde{c}^{\prime}$ are never larger than unity for the parameters considered (see e.g. Fig. 2 in ref.[24]), this explains the relative weakness of the oscillations in fig. 14(a). It also helps to explain the behavior of fig. 14(b) (although in this case one is far from the narrow limit): for a Schulz distribution $\overline{\epsilon^{3}}=2{\overline{\epsilon^{2}}}^{2}$ and hence the correction in eq.(18) vanishes. Accordingly, in a Schulz distribution the shape corrections to $\delta(z)$ will become significant only at large $\delta$. In fact, comparing $\delta(z)$ for $\delta=0.404$ between the Schulz and top hat parents (fig. 14), one can see that for a Schulz the shape of 

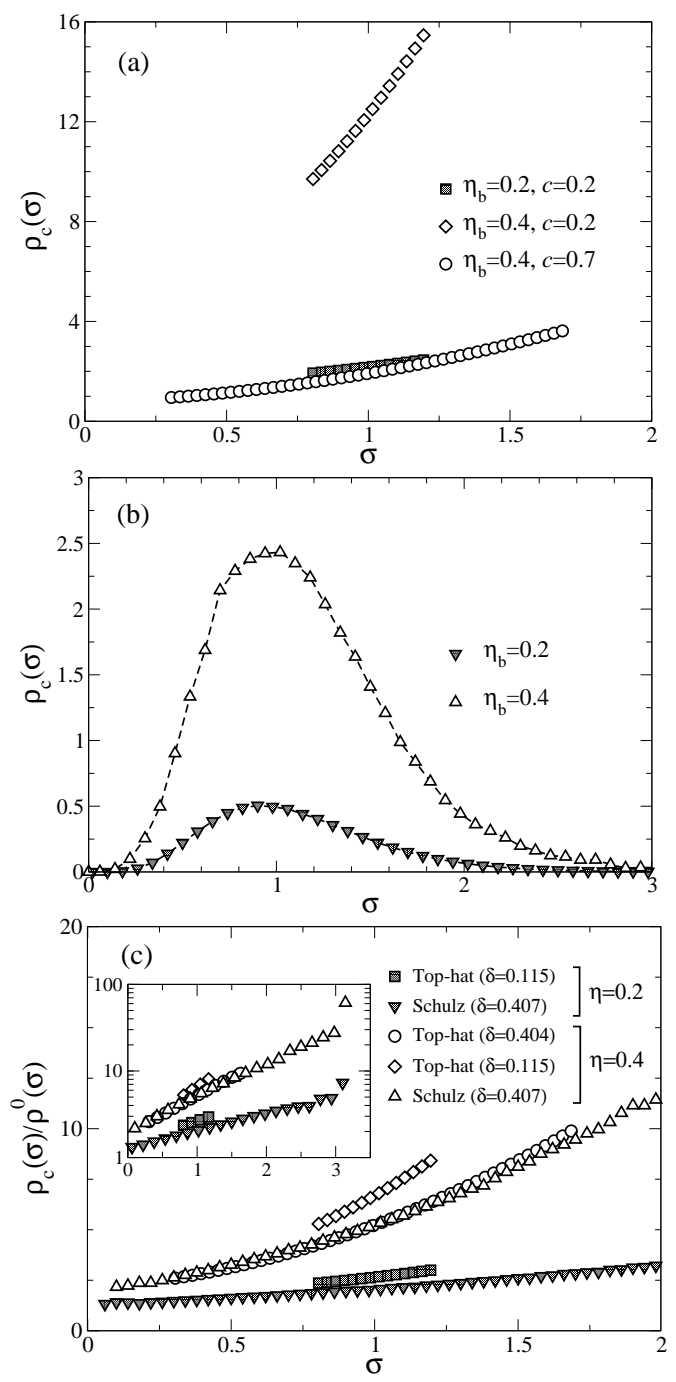

FIG. 7: Contact values $\rho_{c}(\sigma)=\rho(\sigma, z=\sigma / 2)$. (a) Top-hat distributions $(\delta=0.115$ and $\delta=0.404)$ at volume fractions $\eta_{b}=0.2,0.4$. (b) Schulz distribution $(\delta=0.407)$. (c) The contact values relative to the bulk density. In all cases, lines are merely guides to the eye. Error bars are smaller than the symbol sizes.

the local degree of polydispersity follows more closely the density profile of the mean species.

Although we have used a definition of the local polydispersity that generalized the conventional form (eq.3) used to describe bulk parent forms, it is interesting to note that, at least for inhomogeneous systems, a definition of $\delta(z)$ based on the local concentration, $\phi(\sigma, \mathbf{r})$, rather than on the local number density, serves to better illustrate the role of polydispersity effects. Accordingly we form a narrow limit expansion of

$$
\widehat{\delta}(z) \equiv \frac{\left[\int d \sigma(\sigma-\bar{\sigma})^{2} \phi(\sigma, z)\right]^{1 / 2}}{\int d \sigma \sigma \phi(\sigma, z)}
$$
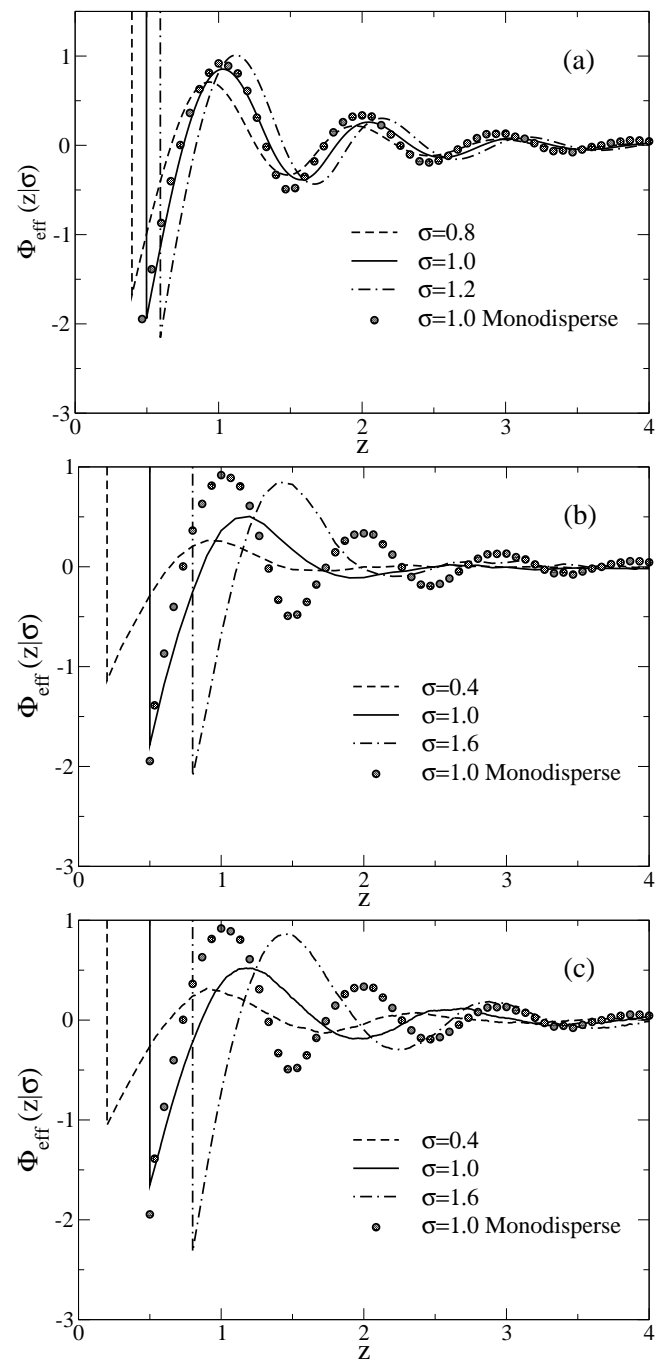

FIG. 8: The effective wall-particle potential at volume fraction $\eta_{b}=0.4$. (a) Narrow top-hat $(\delta=0.115)$; (b) Schulz, $(\delta=0.407)$ and (c) Wide top-hat $(\delta=0.404)$ distribution.

which yields,

$$
\widehat{\delta}(z) \simeq \bar{\sigma} \sqrt{\overline{\epsilon^{2}}}\left\{1+\left[\frac{\overline{\epsilon^{3}}}{2 \overline{\epsilon^{2}}}-\overline{\epsilon^{2}}\right]\left[\tilde{c}^{\prime}(z)-\tilde{c}^{\prime}(\infty)\right]\right\} .
$$

One sees that this last expression does not depend on the local density of the mean species; accordingly we would generically expect a much weaker variation of $\hat{\delta}(z)$ for a Schulz, and for other asymmetric parents. This alternative definition of the local polydispersity thus highlights the fact that in the narrow limit the local segregation of species tends to preserve the width of the parent imposed in the bulk.

\section{DISCUSSION AND CONCLUSIONS}

In summary we have employed specialized MC simulations techniques and DFT to study the effects of a hard 

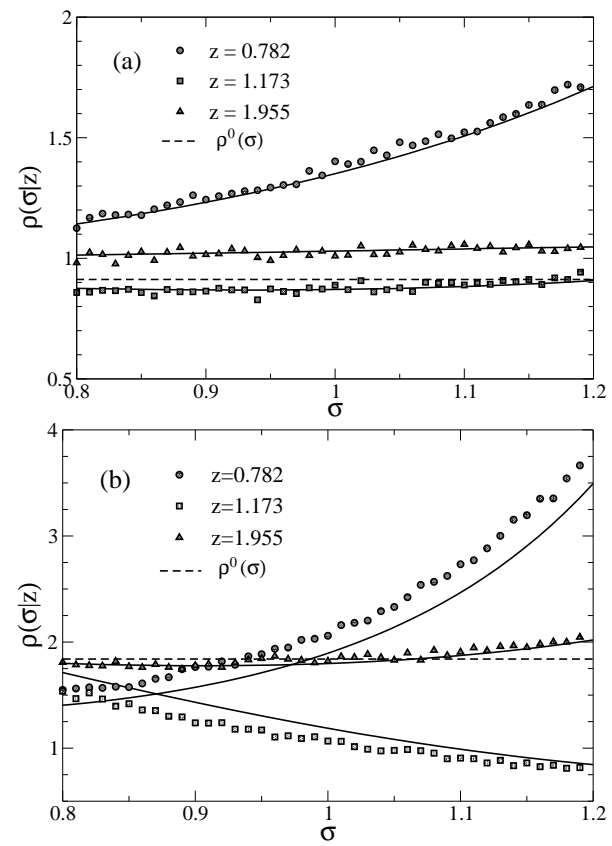

FIG. 9: The size distribution at selected distances $z$ from the wall for the narrow top-hat distribution $(\delta=0.115)$. (a) $\eta_{b}=0.2$, (b) $\eta_{b}=0.4$. Symbols: Monte Carlo results. Solid lines: DFT results.

wall on the properties of polydisperse hard spheres. Bulk phase (parent) distributions of the top-hat and Schulz forms were considered. In the former case, degree of polydispersity $\delta=0.115$ and $\delta=0.404$ were studied, while in the latter case, $\delta=0.407$ was used. In all cases the density distribution $\rho(\sigma, z)$ was obtained and analyzed for bulk volume fractions $\eta_{b}=0.2$ and $\eta_{b}=0.4$.

The original motivation for this study was a desire to gauge the accuracy of the DFT predictions of ref. [24] via a like-for-like comparison with simulation. The results presented in sec. III demonstrate that the agreement is extremely good. Indeed, the DFT calculations provide a quantitatively accurate description of the system properties at $\eta_{b}=0.2$ for both parent forms studied. Even for the higher volume fraction $\eta_{b}=0.4$, the agreement is semi-quantitative. Clearly this finding bodes well for the future utility of DFT in investigating other polydispersity related issues in inhomogeneous fluid.

Beyond this, the present study extends that of ref. [24] by considering the nature of the attractive depletion interactions in the vicinity of the hard wall. Analysis of contact values showed a clear preference for the largest particles to occupy positions in which their surfaces touch the wall. Indeed for the Schulz distribution, the contact values of particles at the cutoff exceeded that in the bulk by over two orders of magnitude. Interestingly, at a given $\eta_{b}$, the degree of contact enhancement as a function of $\sigma$, seems quite insensitive to the distribution shape (cf. fig. $7(\mathrm{c})$ ) for a given $\delta$. This finding was found to extend to the form of the effective potentials (fig. 8) near the wall. Clearly however our comparison is far from
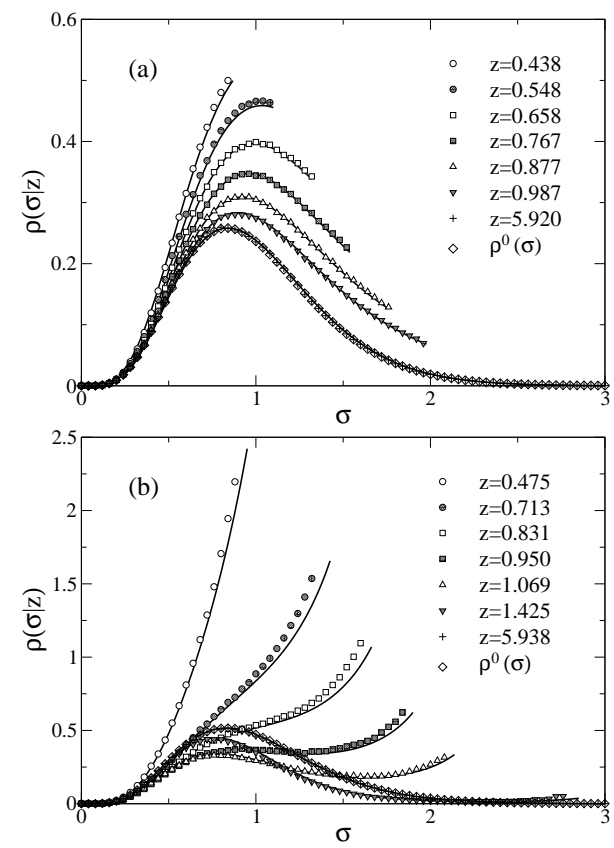

FIG. 10: The size distribution $\rho(\sigma \mid z)$ at selected distances $z$ from the wall for the Schulz distribution $(\delta=0.407)$. (a) $\eta_{b}=0.2$, (b) $\eta_{b}=0.4$. Symbols: Monte Carlo results. Solid lines: DFT results.

exhaustive in this regard and further studies would be required to determine whether this observation applies more generally.

The interplay of the $\sigma$-dependent attractive depletion forces and the geometrical constraint imposed by the wall was found to radically alter the local size distribution. Indeed in the case of the Schulz parent at $\eta_{b}=0.4$, a minimum in $\rho(\sigma \mid z)$ was observed to develop within a certain range of $z$. Another interesting feature, was the appearance of oscillatory structure in the local degree of polydispersity $\delta(z)$. This was accompanied, very close to the wall, by considerable reduction in the local degree of polydispersity with respect to the bulk. It is tempting to speculate that this latter finding may have some bearing on recent experimental observations of wall induced freezing in a polydisperse system of colloidal hard spheres that apparently forms a glass in the bulk [39]. Polydispersity is known to hinder freezing in colloidal systems [6], and consequently any narrowing of $\rho(\sigma)$ due to the presence of a wall may serve to promote the formation of an ordered crystal phase.

With regard to the size segregation effects originally reported in ref. [24], we find that the anti-correlation in the oscillations of the local concentration between the largest and smallest permitted particles is significantly more pronounced for small $\delta$ than for large $\delta$ at a given $\eta_{b}$. Furthermore as for small $\delta$, the period of the oscillations in $z$ appears to be close to that of the mean particle diameter. Both these findings are in accord with the predictions of perturbative calculations [24]. For large $\delta$, by contrast, the present work shows that distribution 

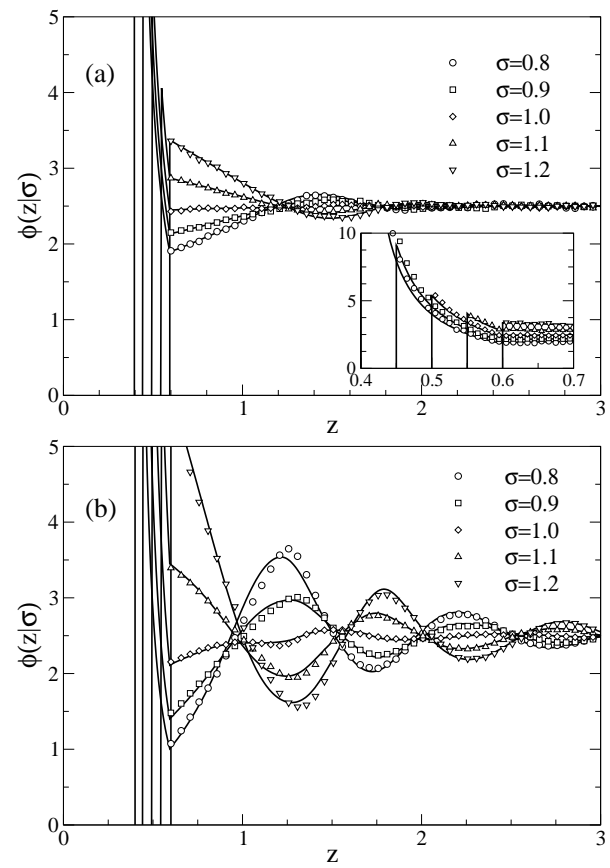

FIG. 11: The local relative concentration $\phi(z \mid \sigma)$ for a narrow top-hat parent distribution $(\delta=0.115)$. Data are shown for a selection of particle sizes $\sigma$. Solid lines are the DFT predictions. (a) $\eta_{b}=0.2$; (b) $\eta_{b}=0.4$.

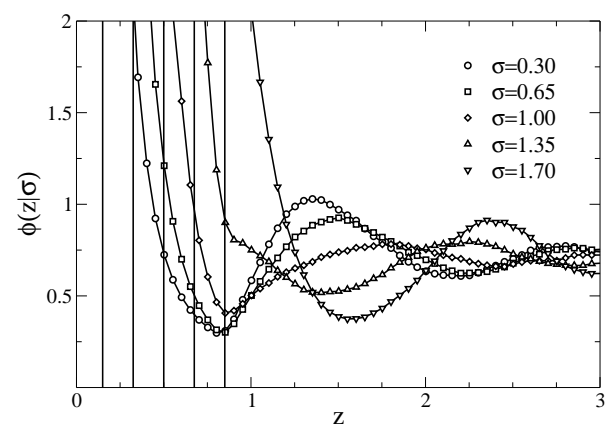

FIG. 12: The local relative concentration for a wide $(\delta=$ $0.404)$ top hat parent distribution at $\eta_{b}=0.4$.

shape effects are important in determining the character of size segregation effects. Thus for the Schulz parent at $\delta \approx 0.4$, the degree of anti-correlation is much less than for the corresponding top-hat parent. Additionally, in the latter case the period of the oscillations appear to be more consistent with the average particle size than for the Schulz distribution. This suggests that it is the presence in the Schulz parent of relatively large higher order moments which leads to this increased disruption of size-segregation effects. Analogous observations apply to the density profiles $\rho(z \mid \sigma)$ themselves. In all cases, (but especially for the Schulz parent), the presence of polydispersity was found to dampen the density oscillations significantly compared to the monodisperse profile $\rho(z)$.

Turning finally to the outlook for further related work,
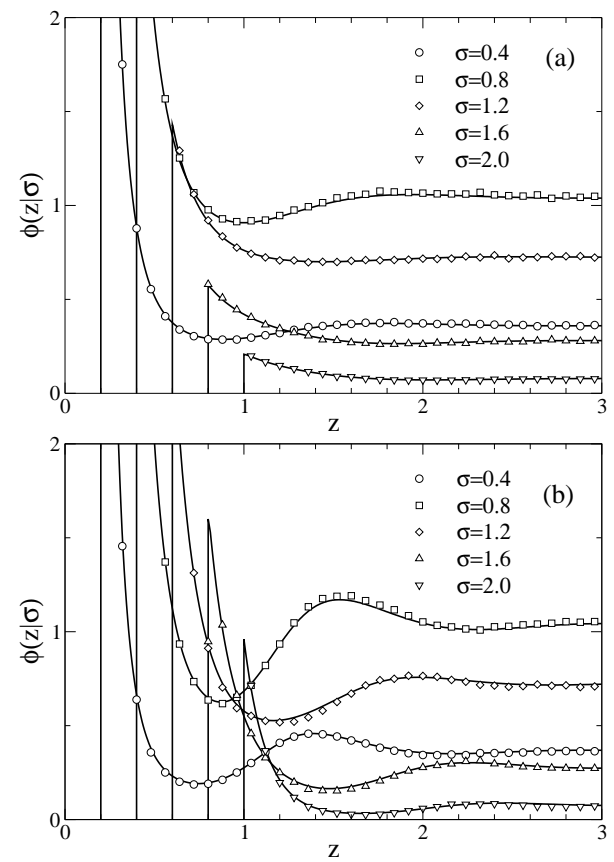

FIG. 13: The local relative concentration $\phi(z \mid \sigma)$ for the Schulz parent $(\delta=0.407)$. Solid lines are the DFT predictions. (a) $\eta_{b}=0.2$; (b) $\eta_{b}=0.4$.
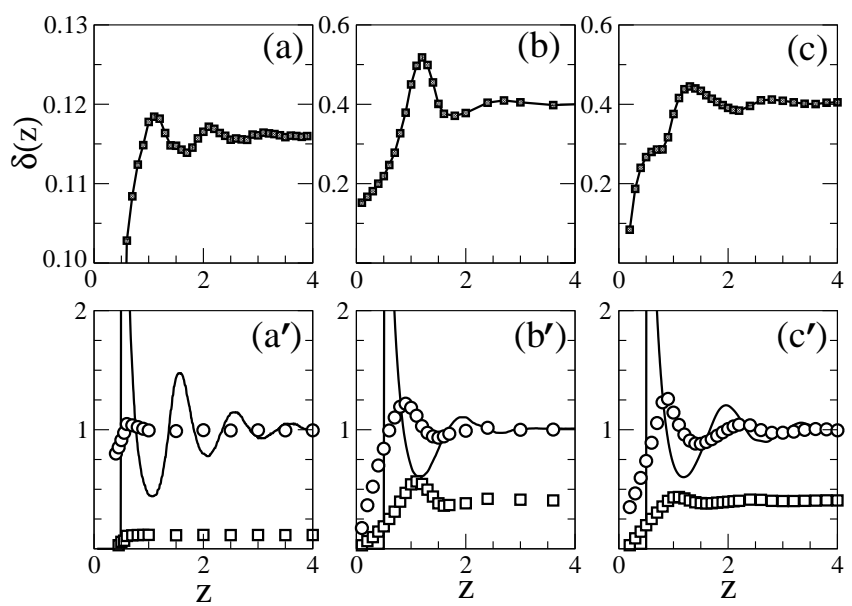

FIG. 14: The spatial dependence of the local degree of polydispersity $\delta(z)$ at volume fraction $\eta_{b}=0.4$. (a) Top-hat, $\delta=0.115$; (b) Schulz $\delta=0.407$; (c) top-hat, $\delta=0.404$. Also shown for the respective parents $\left(\left(\mathbf{a}^{\prime}\right)\right.$ etc $)$ are the mean (circles) and the standard deviation (squares) of the local size distribution $\rho(\sigma \mid z)$. Lines show the profile of the mean species $\rho(z \mid \sigma=1)$, relative to its bulk value.

an interesting open question is whether size segregation effects analogous to those considered here for an inhomogeneous system might also occur in bulk polydisperse fluids i.e. whether pairs of hard spheres of prescribed sizes $\sigma$ and $\sigma^{\prime}$ are preferentially found with certain separations. This could be investigated by appeal to the measured form of the pair distribution function $g\left(r, \sigma, \sigma^{\prime}\right)$. We hope 
to report on this matter in a future publication.

\section{Acknowledgments}

This work was supported by the EPSRC, grant number GR/S59208/01. I.P. acknowledges financial support from DGICYT of the Spanish Government. NBW acknowledges a useful discussion with R. Evans.
[1] See e.g. C.D. Diakoumakos, Q. Xu, F.N. Jones, J. Baghdachi, L.M. Wu, J. Coatings Tech. 72(908) 61 (2000); P.A. Callais and O. Guerret, Eur. Coatings Journal, Aug 2002.

[2] R.G. Larson, The Structure and Rheology of Complex fluids (Oxford University Press, New York, 1999).

[3] See eg. V. Bordereau V, Z.H. Shi, L.A. Utracki, P. Sammut, M. Carrega, Polymer Eng. Science 321846 (1992); D. Nichetti, I. Manas-Zloczower, J. Rheology 42951 (1998).

[4] D.J. Fairhurst and R.M.L. Evans, Colloid \& Polymer Science 282, 766 (2004).

[5] P.D. Kaplan, J.L Rouke, A.G. Yodh, D.J. Pine, Phys. Rev. Lett. 72, 582 (1994) and A.D. Dinsmore, P.B. Warren, W.C.K. Poon, A.G. Yodh, Europhys. Lett. 40, 337 (1997).

[6] P.N. Pusey in Liquids, freezing and glass transition, edited by J.-P. Hansen, D. Levesque and J. Zinn-Justin (North Holland, Amsterdam, 1991).

[7] J.A. Gualtieri, J.M. Kincaid and G. Morrison, J. Chem. Phys. 77, 521 (1982).

[8] J.J. Salacuse and G. Stell, J. Chem. Phys. 77, 3714 (1982).

[9] R.M.L. Evans, Phys. Rev. E59, 3192 (1999)

[10] R.M.L. Evans, J. Chem. Phys. 114, 1915 (2001)

[11] P. Sollich and M.E. Cates, Phys. Rev. Lett. 80, 1365 (1998)

[12] P.B. Warren, Phys. Rev. Lett. 80, 1369 (1998).

[13] P. Sollich, P.B. Warren and M.E. Cates, Adv. Chem. Phys. 116, 265 (2001)

[14] See P. Sollich, J. Phys.: Condens. Matter 14, R79 (2002) for a review of contemporary theoretical approaches to polydisperse phase equilibria.

[15] N.B. Wilding and P. Sollich, Europhys. Lett. (in press); N.B. Wilding, M. Fasolo and P. Sollich. J. Chem. Phys. (in press).

[16] M. Fasolo and P. Sollich, Phys. Rev. Lett. 91, 068301 (2003).

[17] M. Fasolo and P. Sollich, Preprint (cond-mat-0405621).

[18] A. Speranza and P. Sollich, J. Chem. Phys., 117, 5421 (2002).

[19] D. A. Kofke and E.D. Glandt, J. Chem. Phys. 87, 4881 (1987); D.A. Kofke and P.G. Bolhuis, Phys. Rev. E59, 618 (1999).

[20] M.A. Bates and D. Frenkel, J. Chem. Phys. 109, 6193 (1998).

[21] N.B. Wilding and P.Sollich, J. Chem. Phys. 116, 7716 (2002).

[22] Early work assumed that only the mean density varies in space and not the density distribution, see eg. J.L. Barrat and J.-P. Hansen, J. Phys. (Paris) 47, 1547 (1986); R.
McRae and A.D.J. Haymet, J. Chem. Phys. 88, 1114 (1988).

[23] R. Evans, in Fundamentals of Inhomogeneous Fluids, edited by D. Henderson (Dekker, New York, 1992), p. 85.

[24] I. Pagonabarraga, M.E. Cates, G.A. Ackland, Phys. Rev. Lett. 84, 911 (2000).

[25] I. Pagonabarraga and M.E. Cates, Europhys. Lett. 55, 348 (2001).

[26] L. Bellier-Castella, H. Xu and M. Baus, Phys. Rev. E65, 021503 (2002).

[27] The term "parent" is chosen to emphasize that under conditions of confinement, the local distribution may differ from the bulk form from which it derives. Similar effects occur when a polydisperse fluid phase separates into coexisting "daughter" phases whose local distributions differ from that of the parent [14].

[28] R. Evans, J.M. Brader, R. Roth, M. Dijkstra, M. Schmidt and H. Löwen. Phil. Trans. R. Soc. London. A 359, 961 (2001); R. Roth, R. Evans and S. Dietrich, Phys. Rev. E 62, 5360 (2000).

[29] N.B. Wilding, J. Chem. Phys. 119, 12163 (2003).

[30] Y. Rosenfeld, Phys. Rev. Lett. 63, 980 (1989).

[31] E. Kierlik and M.L. Rosinberg, Phys. Rev. A 42, 3382 (1990).

[32] Y. Rosenfeld, M. Schmidt, H. Löwen, P. Tarazona, Phys. Rev. E 55, 4245 (1997); J.A. Cuesta, Y. Martinez-Raton and P. Tarazona, J. Phys. Cond. Matt. 14, 11965 (2002).

[33] See eg. R. Evans in Liquids at interfaces edited by J. Charvolin, J.F. Joanny, and J. Zinn-Justin (NorthHolland, Amsterdam, 1990).

[34] T. Boublik, J. Chem. Phys. 53, 471 (1970).

[35] G.A. Mansoori. N.F. Carnahan, K.E. Starling, T.W. Leland, J. Chem. Phys. 54, 1523 (1971).

[36] B. Götzelmann, R. Roth, S. Dietrich , M. Dijkstra and R. Evans, Europhys. Lett. 47, 398 (1999); R. Roth, R. Evans and S. Dietrich, Phys. Rev. E62, 5360 (2000).

[37] The situation we consider in this work is qualitatively different to that studied by D. Goulding and J.-P. Hansen, Mol. Phys. 99, 865 (2001). These authors investigated the depletion potential between two isolated large spheres immersed in a solvent of polydisperse small spheres.

[38] It is worth stressing that these phase shifts are not simply a consequence of the fact that the centers of smaller particles can approach the wall more closely than larger ones. They remain even if one represents the distance from the wall in terms of a shifted coordinate $z^{*}(\sigma)=z-\sigma / 2$.

[39] R.P.A. Dullens, W.K. Kegel, Phys. Rev. Lett. 92, 195702 (2004). 